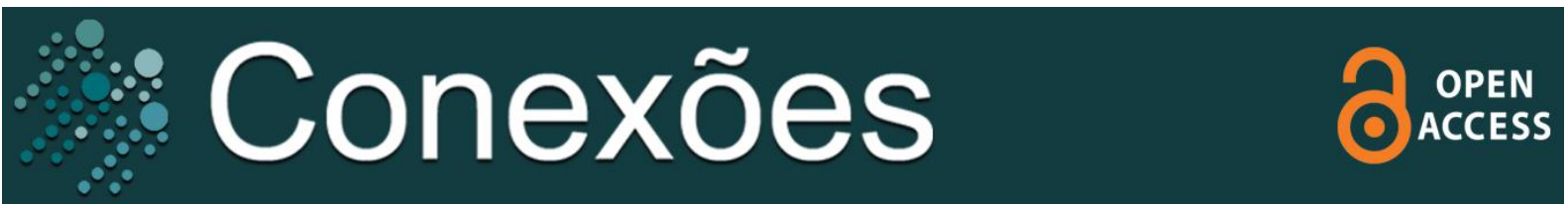

Relato de experiência

\title{
Dialogar con el cuerpo en la clase de Educación Física
}

\author{
Dialogando com o corpo na aula de Educação Física
}

Dialoguing with the body in Physical Education class

\author{
Francisco Javier Giles Girela ${ }^{1}$ \\ Enrique Rivera García ${ }^{1}$ \\ Carmen Trigueros Cervantes ${ }^{1}$
}

\begin{abstract}
RESUMEN
Objetivo: La Tertulia dialógico corporal como metodología de trabajo en el aula de Educación Física es el objeto del artículo. Método: Apoyándonos en las bases del aprendizaje dialógico puesto en acción en las Comunidades de Aprendizaje y sus actuaciones de éxito, hemos creado una adaptación de la tertulia utilizada en ellas para acercar el cuerpo a los niños y niñas de la enseñanza básica, desde posiciones reflexivas y críticas, ayudándoles a construir su corporeidad con el otro desde una perspectiva social. Resultados: Una vez analizadas las bases que sustentan la propuesta, exponemos sus principios y las fases que la desarrollan, analizando posteriormente los aspectos claves de una experiencia llevada a la práctica de aula, desde el análisis de los discursos que niños y niñas construyen en el marco de la tertulia dialógico corporal. Consideraciones finales: Por último, entendemos importante que la educación Física se abra al diálogo y salga de los modelos directivos que la colonizan.
\end{abstract}

Palabras Clave: Educación física escolar. Innovación pedagógica. Aprendizaje dialógica.

\footnotetext{
1 Universidad de Granada, Faculdade Ciencias de la Educación, Grupo de Investigación: Educación física y transformación social, Granada, España.

\section{Correspondência:}

Francisco Javier Giles Girela. Facultad de Ciencias de la Educación, Campus Universitario de la Cartuja, 18071 - Granada, Espanha. Email: javiggr@hotmail.com
} 


\section{RESUMO}

Objetivo: Discussão Corporal Dialógica como metodologia de trabalho na sala de aula de Educação Física é o tema deste artigo. Método: Com base nos fundamentos da aprendizagem dialógica colocada em ação nas Comunidades de Aprendizagem e suas ações bem sucedidas, criamos uma adaptação do talk show utilizado nelas para aproximar o corpo das crianças da educação básica, a partir de posições reflexivas e críticas, ajudandoas a construir sua corporeidade com o outro a partir de uma perspectiva social. Resultados: Uma vez analisadas as bases que sustentam a proposta, explicaremos seus fundamentos e as fases que a desenvolvem, analisando posteriormente os aspectos-chave de uma experiência realizada em sala de aula, a partir da análise dos discursos que as crianças constroem no âmbito da discussão do diálogo corporal. Considerações Finais: Por último, entendemos importante que a Educação Física se abra ao diálogo e questione os modelos diretivos que a colonizam.

Palavras-chave: Educação física escolar. Inovação pedagógica. Aprendizagem dialógica.

\section{ABStRACT}

Objective: The Body Dialogical Gathering as a working methodology in the Physical Education classroom is the object of the article. Method: Relying on the basis of dialogical learning put into action in the Learning Communities and their successful performances, we have created an adaptation of the discussion group used in them to bring the body closer to the children of basic education, from reflective and critical positions, helping them to build their corporeality with the other from a social perspective. Results: Once analyzed the bases that sustain the proposal, we expose its bases and the phases that develop it, analyzing later the key aspects of an experience taken to the practice of classroom, from the analysis of the speeches that children construct in the frame of the corporal dialogic tertulia. Final considerations: Finally, we understand that Physical education teachers may dialogue and leave the directive models that colonize it.

Keywords: School physical education. Pedagogical innovation. Dialogical learning. 


\section{INTRODUCCIÓN}

Vamos a empezar el relato de esta experiencia partiendo de los retos que identifica Devís-Devís (2018) para poder dotar de sentido a la Educación Física en el actual curriculum educativo. En primer lugar, apuesta por la incorporación del conocimiento teórico a la asignatura sin correr el riesgo de caer en su academización; al contrario, buscar desde su incorporación la creación de una conciencia crítica en el alumnado. Como segunda clave plantea el dotar de significado a lo que enseñamos y pueda ser transferible a la vida cotidiana, entendida en toda su amplitud, facilitando la creación de sentimientos de pertenencia social a un grupo. Además, apuesta por una Educación Física que se apoye en los aspectos valóricos que construyen ciudadanía y permiten la aceptación de la diversidad en todas sus manifestaciones. Por último, Devís-Devís (2018) apuesta por un maridaje entre universidad y escuela. Que los docentes de la universidad bajen de sus torres de marfil donde construyen conocimiento teórico basado en hipótesis y se vinculen con los docentes de los niveles básicos y medios y busquen, desde la investigación colaborativa, soluciones a los problemas reales de la escuela y la Educación Física que actualmente la coloniza.

El reto a los planteamientos anteriores pasa por una necesaria revisión de conceptos claves, especialmente relacionados con el cuerpo y sus posibilidades de diálogo consigo mismo, con los demás y con el contexto que habitan. Además, tendremos que ver cuáles son las funciones actuales que debe cubrir la Educación Física Escolar como parte de la cultura a la que pertenece y superar los modelos de aprendizaje predominantes en la escuela actual, para incorporar el aprendizaje dialógico como estructura básica, que nos puede facilitar la construcción de una renovada Educación Física que encuentre su espacio en el currículum escolar.

Centrándonos en el cuerpo y siguiendo a Turner, (1994) para entender el significado del cuerpo dentro de la teoría social debemos contextualizarlo en el actual marco social, muy decantado a una economía capitalista basada en el consumo compulsivo, el fuerte avance del postmodernismo en los 90 y la primera década del siglo XXI, los nuevos paradigmas emergentes, especialmente el centrado en las teorías feministas y como ya adelantaba hace cuarenta años Foucault, citado por Turner (1994), el fuerte impacto de la biopolítica en la Sociedad con los cambios demográficos a nivel mundial, la imparable globalización y asentamiento del pensamiento único, el envejecimiento poblacional en la sociedad occidentaliza, la pérdida de la confianza en el poder político y el consiguiente avance del poder económico que dicta las pautas sociales a seguir. Sin olvidar la emergencia provocada por las nuevas pandemias como la crisis del SIDA y el actual COVID-19, que están provocando una nueva visión del cuerpo en el ámbito social.

Dentro de este marco social, se impone un modelo de cuerpo multifacético en el que emergen una serie de ejes claves que lo definen: salud, estética, placer, 
predominando una mirada hedonista del mismo (MARTÍNEZ-ÁLVAREZ, 2013). Lógicamente la Educación Física no puede quedar al margen de esta visión y debe, por una parte, tomar conciencia de dónde se sitúan nuestros alumnos y alumnas para nada inmunes a estos aspectos y por otro lado, crear una conciencia crítica que les haga tomar conciencia de los riesgos ante los que se enfrentan (ÁGUILA; LÓPEZ, 2019). En palabras de (BARBERO, 2005), debe ser la Educación Física quien otorgue legitimidad a la educación del cuerpo en la escuela; su comprensión y vivencia desde perspectivas más críticas y dialógicas, les ayudará a lograr un capital corporal que les independice y aleje de la cultura y visiones dominantes que, sobre el cuerpo, están tratando de homogeneizar a nuestra sociedad occidentalizada. Lograr que giren su mirada hacia planteamientos abiertos a la subjetividad de los cuerpos, su construcción con y desde el otro, que sepan mirar intra antes de proyectarse extra. Abiertos hacia planteamientos que logren la descolonización eurocéntrica y norteamericana a la que estamos sometidos, recuperando prácticas corporales más cercanas a nuestra cultura; a planteamientos que nos alejen del dualismo cartesiano y nos acerquen al cuerpo tal y como planteaba Merleau-Ponty, citado por (CARRASCO-SEGOVIA; HERNÁNDEZ-HERNÁNDEZ, 2020), desde una perspectiva experiencial y creativa que nos ayude a la implicación social y la construcción con el otro desde las experiencias encarnadas (RODRÍGUEZ, 2014).

Esta nueva visión de la corporeidad, alejada de las clásicas funciones otorgadas a la Educación Física encaminadas básicamente al mantenimiento de la salud y dotar de herramientas para construir un ocio activo desde en la práctica de la actividad física, tenía sentido en un contexto social donde los sujetos, especialmente los provenientes de las clases obreras, debían ejercer su actividad laboral a pleno rendimiento en las fábricas, al tiempo que defender de sus enemigos a su "patria" -concepto sustituible por los intereses económicos del capital-. Este enfoque de la Educación Física dentro del currículum escolar se construye hace más de cien años, apoyándose en dos pilares: el deporte y la gimnasia, es decir se ancla en un paradigma biomotriz que mantiene y potencia el dualismo cartesiano al que hacíamos anteriormente referencia. Estamos ante un enfoque predominante, si no curricularmente, si en la práctica, y que se sigue visibilizando cada día en la mayoría de los centros educativos, siendo la excepción aquellos en los que se comienza a abrir una ventana a enfoques donde la corporeidad y la construcción social del cuerpo comienzan a estar presentes. Tratando de sintetizar las funciones que la Educación Física debiera asumir para el siglo XXI y siguiendo a (DEVÍS-DEVÍS; MOLINA, 2004), la primera debiera estar vinculada a la justicia social, especialmente en su vertiente de respeto a la diversidad y compensación de las desigualdades sociales; para su logro, tendremos que potenciar entre el profesorado y los estudiantes de los centros de formación la idea de reconstruir el conocimiento y las experiencias, potenciando una doble mirada social y educativa. 
Si estamos posicionados en esta dirección comenzaremos a ver a la Educación Física como espacio abierto para el diálogo, donde nuestro alumnado sea capaz de tomar conciencia de la cultura que se transmite, al tiempo que abre la mirada a la realidad vivida en sus prácticas corporales cotidianas. Es aquí donde cobra fuerza y entronca el aprendizaje dialógico con otra forma de hacer la asignatura (MORENO-DOÑA; CAMPOS-VIDAL; ALMONACID-FIERRO, 2012). EI problema que se nos plantean es cómo llevar a los centros educativos este enfoque de la Educación Física. Aprender desde el otro, construir los aprendizajes a partir de nuestra experiencia, transmitir la cultura social relevante y dialogar desde la horizontalidad.

Si entramos en el último eje de nuestro trabajo, el aprendizaje dialógico, podemos observar que en el campo educativo se está produciendo un giro hacia un aprendizaje basado en la bidireccionalidad, abandonando las prácticas del aprendizaje centradas en la unidireccionalidad. Esto está facilitando la entrada del diálogo al aula y lo que ello conlleva en cuanto al potenciamiento de las relaciones de igualdad y la aparición de otras formas de aprender que tratan de hacer confluir la cultura académica con las experiencial (AUBER; GARCIA; RACIONERO, 2009). Estamos asistiendo al renacimiento de teorías que potencian esta línea de actuación en las aulas: teoría de la acción dialógica (Freire, 1970) o la teoría de la acción comunicativa (HABERMAS, 1987) pueden ser un excelente ejemplo. Vemos cómo la persona entendida como ser dialógico que aprende en contextos de participación con los demás, adquiere sus aprendizajes al tiempo que mejoran la convivencia al emerger el respeto al otro y a la diversidad que se presenta en las aulas (FLECHA, 2009).

El aprendizaje dialógico se apoya en siete principios básicos: dialogar desde la horizontalidad; potenciar la inteligencia desde la perspectiva académica y la práctica; ayudar a las personas en su transformación, tanto personal como social; facilitar la formación instrumental, sin renunciar a los aspectos valóricos y democráticos; dotar de sentido y utilidad al aprendizaje; buscar siempre la solidaridad, especialmente con las personas más desfavorecidas y excluidas y potenciar la diversidad desde la exaltación de la diferencia como positivo (AUBERT et al., 2009).

Pero el reto no está en definir las claves de un buen aprendizaje dialógico, lo vamos a encontrar en el cómo llevarlo al espacio del aula para generar actuaciones de éxito. En este artículo abordamos una sola estrategia de las planteadas en las Comunidades de Aprendizaje: grupos interactivos, tertulias dialógicas, la formación de las familias y otros agentes comunitarios, la participación educativa de la comunidad y el modelo dialógico de resolución de conflictos (FERREYRA, 2016). En la Educación Física Escolar tenemos actualmente alguna referencia de haber utilizado estrategias como la resolución de conflictos desde el modelo dialógico (CAPLLONCH; FIGUERAS; CASTRO, 2018) o los grupos interactivos (CASTRO-SANDÚA; GÓMEZ-GONZÁLEZ; MACAZAGA-LÓPEZ， 2014; GILES- 
GIRELA; RIVERA-GARCÍA; TRIGUEROS-CERVANTES, 2020). Pero no hay referencias de la utilización de la tertulia dialógica salvo la experiencia que narraremos más adelante y de la que se hizo una puesta en práctica en centros de Comunidades de Aprendizaje (RIVERA-GARCÍA; SÁNCHEZ-LÓPEZ; GILES-GIRELA, 2018).

La tertulia dialógica orientada al aula, tal y como es contemplada en las Comunidades de Aprendizaje, se organiza en modo de encuentro para dialogar en torno a un tema que puede pertenecer a los diferentes campos del saber: literatura, música, artes plásticas, etc. (FERREYRA, 2016). El objetivo de la tertulia se encuentra en que cada niño o niña exprese de forma descriptiva, reflexiva y crítica su opinión sobre el tema central de la tertulia. El papel del docente es fundamentalmente de dinamizador, fomentando la participación de todos y todas. Habitualmente se inicia con la exposición por cada uno de los participantes del fragmento que le ha llamado la atención del material trabajado previamente (literario, musical, icónico, auditivo o visual) y el porqué de su elección (SERRANO; MIRCEVA; LARENA, 2010).

La tertulia dialógica lo que pretende es favorecer el aprendizaje entre iguales, fomentando su construcción con el otro y su transformación tanto en el ámbito social, como instrumental y valórico. Estamos ante un auténtico facilitador del cambio y la innovación en el ámbito escolar, perfectamente transferible y realizable a cualquier nivel, ya que podemos plantearla tanto en el espacio de la educación infantil como en el universitario, pasando por todos los niveles intermedios.

El objeto de este artículo es plantear la adaptación que nosotros hacemos de las tertulias dialógicas al ámbito corporal. Entendemos que el cuerpo debe ser el eje en torno al que hacemos pivotar la propuesta y desde esta mirada, nuestro objetivo no puede quedar reducido al ámbito cognitivo y o valórico; debemos ir más allá y ser capaces de dialogar con la palabra y con el cuerpo con la finalidad de enriquecer nuestra corporeidad.

\section{MÉtodo de tRABAjo}

Nuestra propuesta no se ajusta en sí misma a ninguna metodología científica, nuestro objetivo, como adelantábamos anteriormente, se asienta en explicar cómo estamos utilizando las tertulias dialógico corporales en el aula de Educación Física. Es por esto por lo que en este espacio hablaremos de la metodología de implementación y no de método científico tal y como lo entendemos, aunque posteriormente, describiremos la evaluación de una propuesta llevada a la práctica en un centro de educación básica.

\section{CONTEXTUALIZANDO LA TERTULIA DIALÓGICO-CORPORAL}


La propuesta pedagógica que guía nuestro hacer en las aulas de Educación Física de las Comunidades de Aprendizaje, está mediada por el aprendizaje dialógico explicado anteriormente. Desde el respeto a sus principios, pero al mismo tiempo buscando su aplicación a las características de nuestra materia, hemos construido un modelo de pedagogía dialógica corporal para el aula de Educación Física (GILES-GIRELA; RIVERA-GARCÍA; TRIGUEROS-CERVANTES, 2020), en el que se incluye como última fase la Tertulia dialógico-corporal (FIGURA 1).

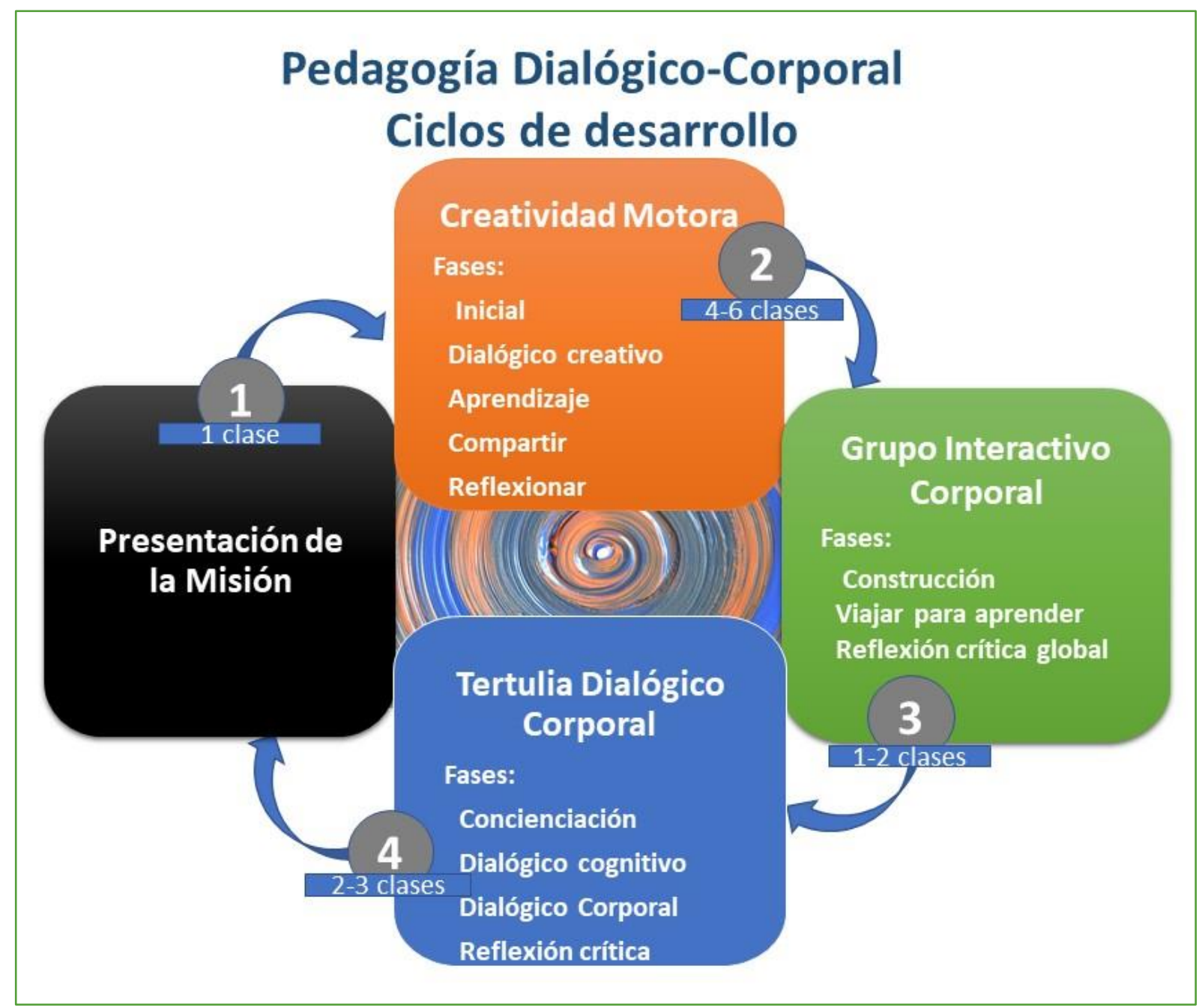

Figura 1 - Fases de la pedagogía dialógico-corporal.

Si queremos hacer mención a que el modelo presentado no es cerrado, es una propuesta que puede ser cíclica, provocandose desde la Tertulia dialógicocorporal la siguiente misión a desarrollar, que no deja de ser nada más que la temática en torno a la que vamos a trabajar en la clase de Educación Física. Como se puede obervar tiene un claro paralelismo con la propuesta metodológica que se realiza en las Comunidades de Aprendizaje, especialmente en la utilización de dos actuaciones de éxito: los grupos interactivos y la tertulia dialógico-corporal. Nuestro objetivo en este artículo se centra en esta última, por lo que si se desea profundizar en el modelo recomendamos la revisión de la literatura que hemos 
generado en torno a él (GILES-GIRELA; RIVERA-GARCÍA; TRIGUEROSCERVANTES, 2020; RIVERA-GARCÍA; SÁNCHEZ-LÓPEZ; GILES-GIRELA, 2018).

\section{Definiendo la Tertulia Dialógico-coporal}

La entendemos como un espacio de aprendizaje dialógico, en el que, partiendo de sus principios, queremos generar en nuestro alumnado aprendizajes relaccionados con el ámbito cognitivo, valórico y motriz. Pretendemos que el aula de Educación Física se transforme en un territorio de aprendizaje global, donde la reflexión y la crítica esten presentes, así como la creatividad de los estudiantes que, trabajando en colaboración, sean capaces de construir sus propias propuestas de aprendizaje motriz en el aula.

Vamos a diferenciar metodológicamente diferentes fases para su implementación en la clase de Educación Física, pudiendo ser la culminación de una propuesta de aprendizaje basada en la pedagogía dialógico-corporal o ser el inicio de una nueva, tal como se mencionaba anteriormente. Por esta razón, cuando anteriormente veíamos en la figura 1 las fases de la pedagogía dialógicocorporal, recordábamos que no debíamos entenderla como lineal, muy al contrario, su planteamiento podría perfectamente asumir un desarrollo secuencial en espiral, contemplando diferentes ciclos a lo largo del proceso de aprendizaje (FIGURA 1).

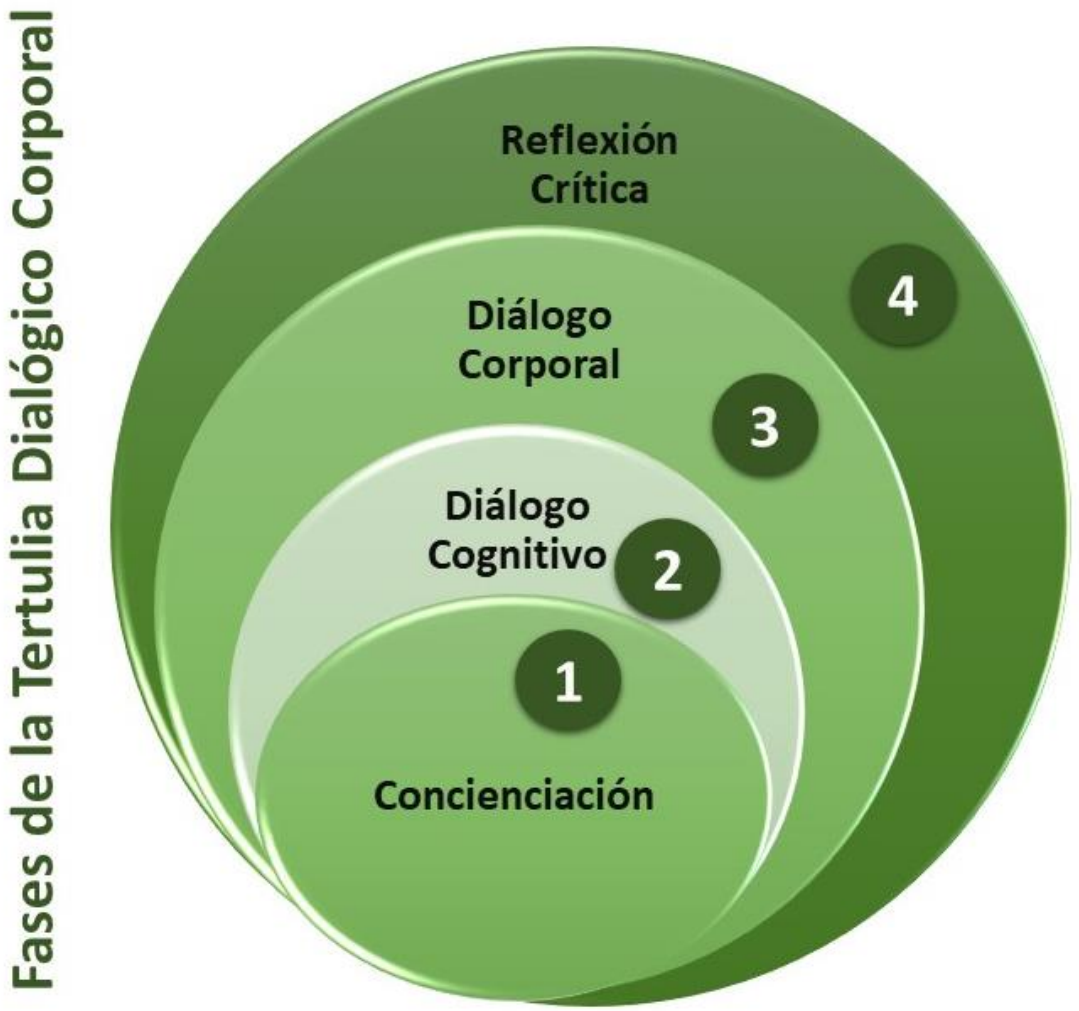

Figura 2 - Fases de la Tertulia Dialógico Corporal. 
Centrándonos en el ciclo de la Tertulia dialógico corporal podemos identifiar cuatro fases o momentos en su implementación en el aula: concienciación, diálogo cognitivo, diálogo corporal y reflexión crítica (Figura 2).

\section{FASE DE CONCIENCIACIÓN}

En ella, nuestra intención es provocar la disonancia cognitiva en el alumnado. Aquí entra en juego el detonante que va a ser el provocador de la posterior reflexión y análisis crítico. Su puesta en acción puede partir mediante la utilización de diferentes estrategias para presentar el objetivo pretendido con la tertulia. Puede ser desde una lectura, una fotografía o un audiovisual. La idea es provocar en nuestros alumnos y alumnas la necesidad de dialogar para aprender con los demás desde lo visto o leído. En una propuesta elaborada para trabajar en clase, nuestro punto de partida fue el respeto a la diverdad del aula, más concretamente nuestro objetivo se centraba en la concienciación empática a partir de los sentimientos y sufrimientos que podría sentir un compañero o compañera que estuviera sufriendo bulling en el aula (RODRÍGUEZ-PALACÍN, 2020). Para ello utilizamos un corto sobre bulling escolar "cobardes" (FERNÁNDEZ-ENGLER, 2017) de cinco minutos de duración. Lo ideal es que el vídeo sea visualizado en la casa y el alumno o la alumna selecciones esa escena o escenas que más le hayan llamado la atención, escribiendo una breve narración de la misma y su opinión sobre lo visualizado en ellas. Una vez en el aula, podemos comenzar con la visualización del corto para pasar posteriormente a la segunda fase del ciclo. Si el contexto social vemos que puede ser un impedimento para que los estudiantes realicen la visualización, podemos dejar este momento para el aula, aunque lógicamente nos quedaríamos con poco tiempo para poner en marcha la siguiente fase.

\section{DiÁlogo COGNITIVO}

Se produce desde la puesta en común de las reflexiones realizadas por los estudiantes. Es el momento de aprender a escuchar, a respetar al otro y entrar en el diálogo sobre aquellos aspectos más relevantes de la temática que estemos trabajando. El docente debe asumir un papel de mediador, animando a la participación y planteando cuestiones y preguntas que nazcan de las reflexiones y críticas de los estudiantes. Debemos intentar que todas y todos participen en el diálogo, para que vayan construyendo su aprendizaje desde los argumentos expuestos en el debate.

\section{DIÁlOGO CORPORAL}

No podemos perder de vista que el objeto de la Educación Física en el currículum escolar es el cuerpo entendido como globalidad. Pero la posibilidad de dialogar con él pasa por dotarle de unas capacidades que le hagan más facil su comunicación con uno mismo, con los demás y el entorno que nos acoge. Por esta 
razón entendemos que debemos superar el nivel de aprendizaje cognitivo para pasar al corporal, en el que nuestros alumnos sean capaces de expresar desde la motricidad y su cuerpo lo que anteriormente han sabido plasmar desde el diálogo verbal.

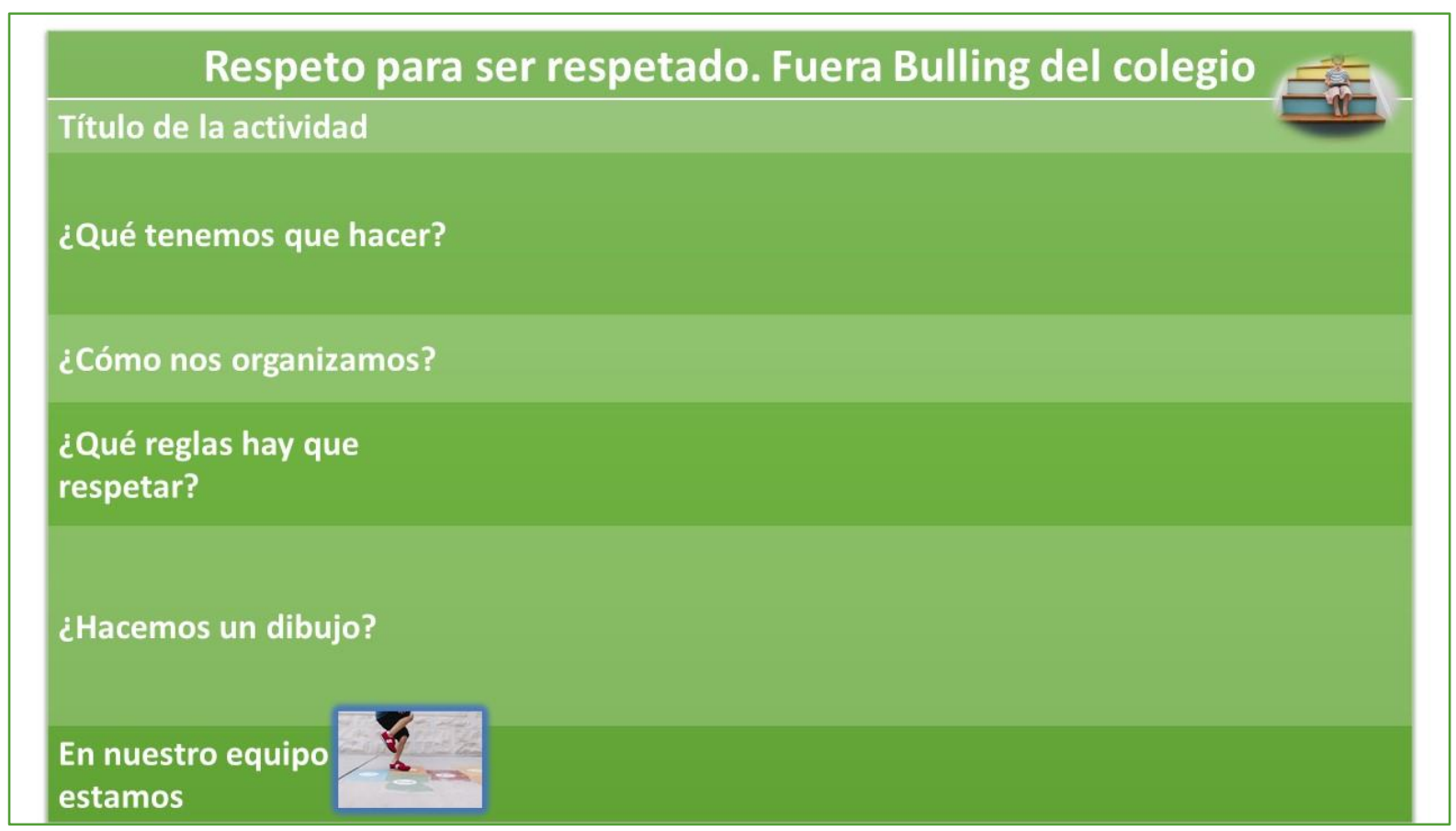

Figura 3 - Hoja de tarea para la construcción de la actividad motriz.

En esta fase, organizaremos a los estudiantes en diversos subgrupos de 6 a 8 personas. El objetivo será que cada uno de los subgrupos realice una propuesta que implique el compromiso de poner en acción sus recursos motrices para lograr el objetivo marcado. En este caso tomar conciencia de las situaciones de bulling que se pueden producir en el centro educativo y cómo actuar frente a ellas de forma preventiva. Una vez preparada la propuesta (FIGURA 3), esta será puesta en práctica con todo el grupo de clase. Recomendamos que, para que funcione la puesta en acción, se utilice una estrategia de circuito, integrando en cada uno de los subgrupos que inician en una estación, representates de cada uno de los equpos iniciales que originariamente han elaborado las diferentes actividades motrices a poner en práctica. Esta estrategia facilita que la explicación inicial, así como el desarrollo de la actividad sea responsabilidad de los estudiantes que la han elaborado.

\section{REFLEXIÓN CRÍtICA}

El punto final de la tertulia dialógica se concentra en esta fase. Es el momento en el que vamos a provocar la reflexión sobre las actividades motrices realizadas ayudando a que se cierre el aprendizaje dialógico sobre la temática realizada. Al mismo tiempo, podremos provocar, si es nuestra intención iniciar desde la propuesta una segunda misión y comenzar el ciclo de la pedagogía dialógico corporal, la emergencia de nuevas temáticas a llevar al espacio de la clase. Para 
facilitar esta fase, partimos de una hoja de evaluación por grupo (FIGURA 4), en la que ayudamos al alumnado a realizar una valoración correcta de las propuestas realizadas.

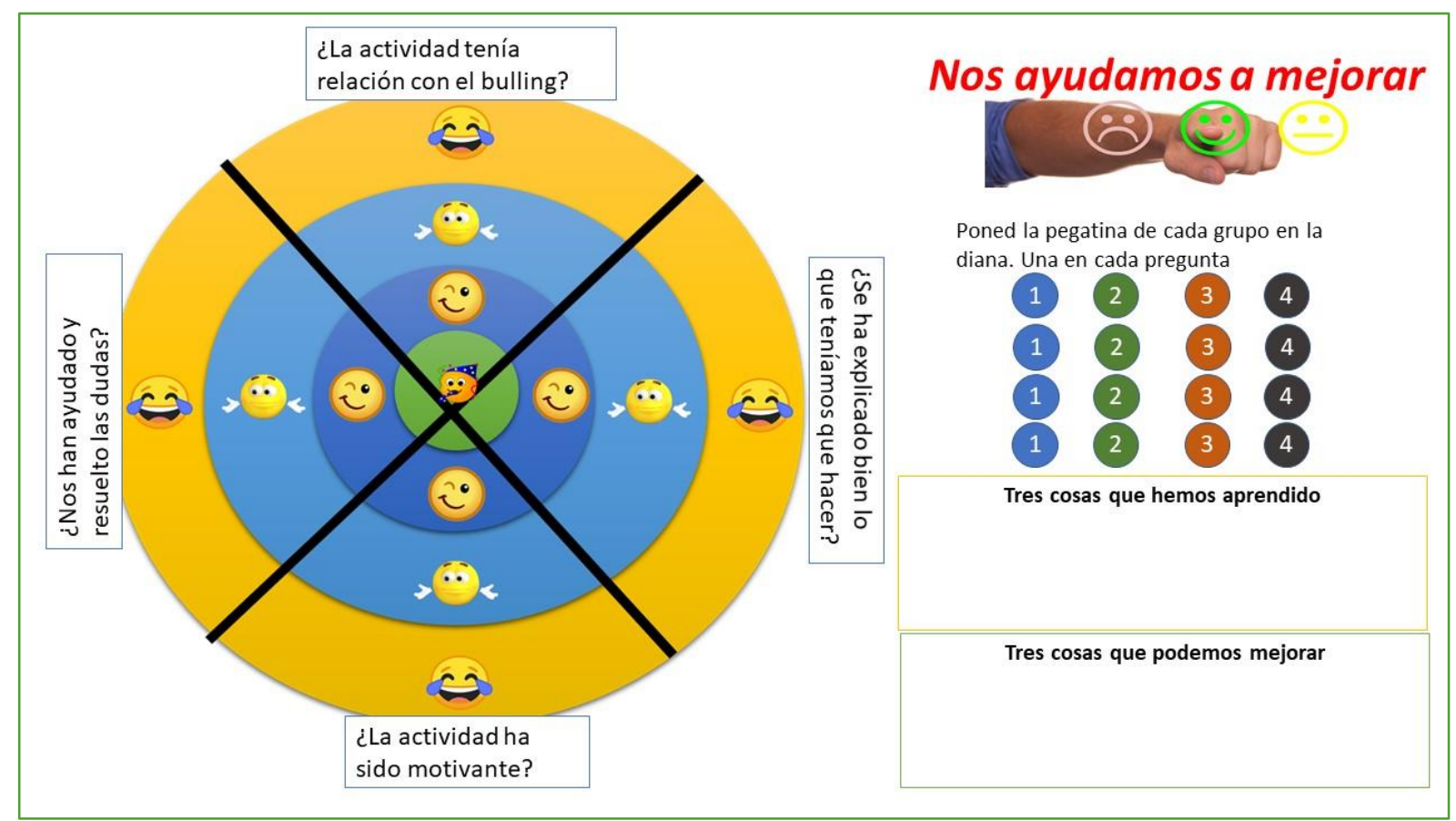

Figura 4 - "diana para mejorar". Hoja de evaluación por grupos.

Es un momento ideal para que los estudiantes aprendan a dialogar, a respetarse, a valorar la honestidad y la justicia y especialmente a comprender que evaluar debe ser dialogar, confrontar puntos de vista diferentes, aprender a argumentar, empatizar con el otro y descubrir que se aprende desde el reconocimiento de nuestras limitaciones y aceptando la ayuda del otro.

\section{DESCRIPCIÓN E INTERPRETACIÓN DE LOS RESULTADOS DE UNA EXPERIENCIA}

La puesta en escena de la tertulia dialógico corporal la realizamos con una experiencia que quedó plasmada en una tesis de grado (SÁNCHEZ-LÓPEZ, 2017) y una publicación posterior realizada a partir de la exposición de la experiencia desarrollada en un Congreso Educativo (RIVERA-GARCÍA; SÁNCHEZ-LÓPEZ; GILES-GIRELA, 2018).

Nuestro objetivo era analizar el discurso del alumnado generado a partir de la visualización de un incidente crítico ocurrido durante el desarrollo de un partido de futbol de categoría infantil. Lo que se estaba poniendo encima de la mesa es el tema de las actitudes negativas que genera el deporte en general y el fútbol en 
particular, cuando no se sabe canalizar desde una vía educativa, poniendo el resultado por encima del proceso vivido.

La experiencia se llevó a cabo en dos centros de enseñanza básica que trabajan bajo el enfoque de las Comunidades de Aprendizaje, donde la tertulia dialógica es una herramienta habitual en sus aulas, pero el enfoque desde la tertulia dialógico corporal era totalmente novedoso. Los contextos sociales de ambos centros son de atención preferente, dado el alto riesgo de exclusión social que presenta el alumnado, al provenir de familias con bajo nivel cultural, social y económico, muchas de ellas migrantes en búsqueda de un futuro que perciben como más favorable para ellos y sus hijos e hijas. En total se realizaron seis tertulias con una presencia de alumnado en cada una de ellas que oscilaba entre las 15 y 25 personas por tertulia, lo que nos ha permitido contar con el discurso de cerca de 120 niños y niñas.

Para la fase de concienciación se utilizó un video al que pusimos por título ¿juego limpio...? . Este audiovisual fue montado exprofeso para la realización de esta tertulia. En esta experiencia se realizó la segunda fase del diálogo cognitivo, objeto de esta investigación, dejando sin realizar las fases de diálogo corporal y reflexión crítica.

El análisis de la información producida (discurso del alumnado) se realizó a partir de la transcripción de cada una de las tertulias, para pasar las mismas al software NVivo (TRIGUEROS-CERVANTES; RIVERA-GARCÍA; RIVERA-TRIGUEROS, 2017) y comenzar el análisis de la información. El punto de partida fue la categorización de la información de forma emergente, creando categorías vivas que, posteriormente, desde la categorización axial fueron construyendo un sistema de categorías del cual partimos para ir concretando el análisis de cada una de ellas. En la figura 5 podemos ver el sistema de categorías final, tal y como quedó una vez realizada toda la categorización emergente y reorganizando las principales categorías en base a las centrales que se identifican (Actitudes negativas, actitudes positivas, familia y medidas a tomar). 


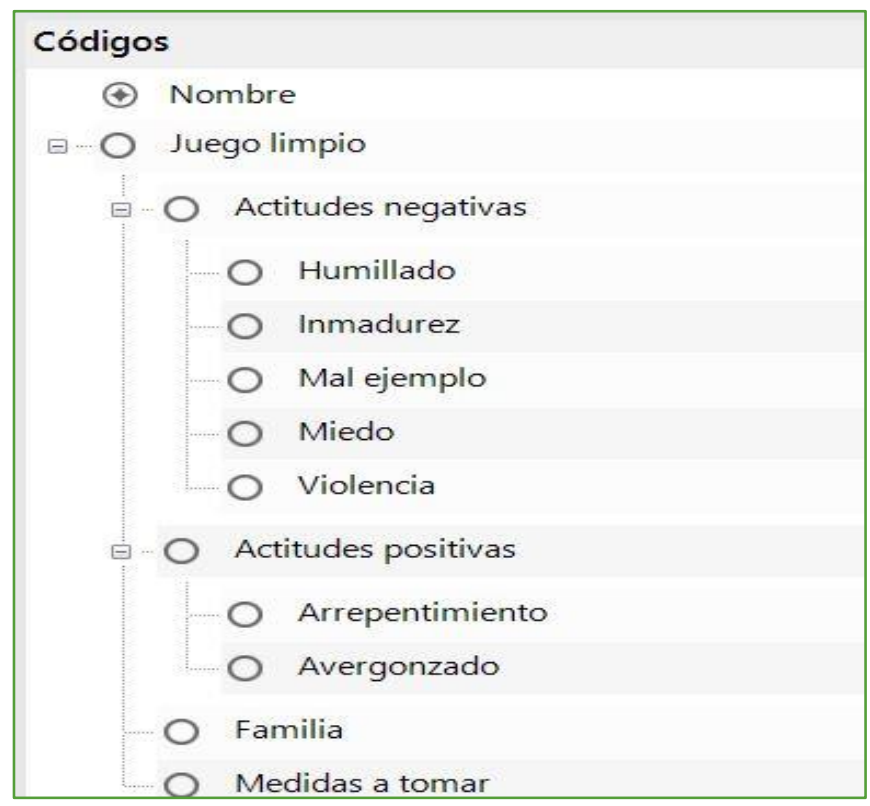

Figura 5 - Sistema axial de categorías.

Para tomar conciencia de los conceptos claves que los niños y niñas utilizan para describir sus sentimientos y opiniones en cada una de las categorías centrales, hemos iniciado el análisis desde una comparativa de nubes de palabras (FIGURA 6) en la que podemos ver, además de la diferencia de conceptos utilizados en cada una de ella, la densidad de estos y la importancia otorgada a cada uno de ellos.

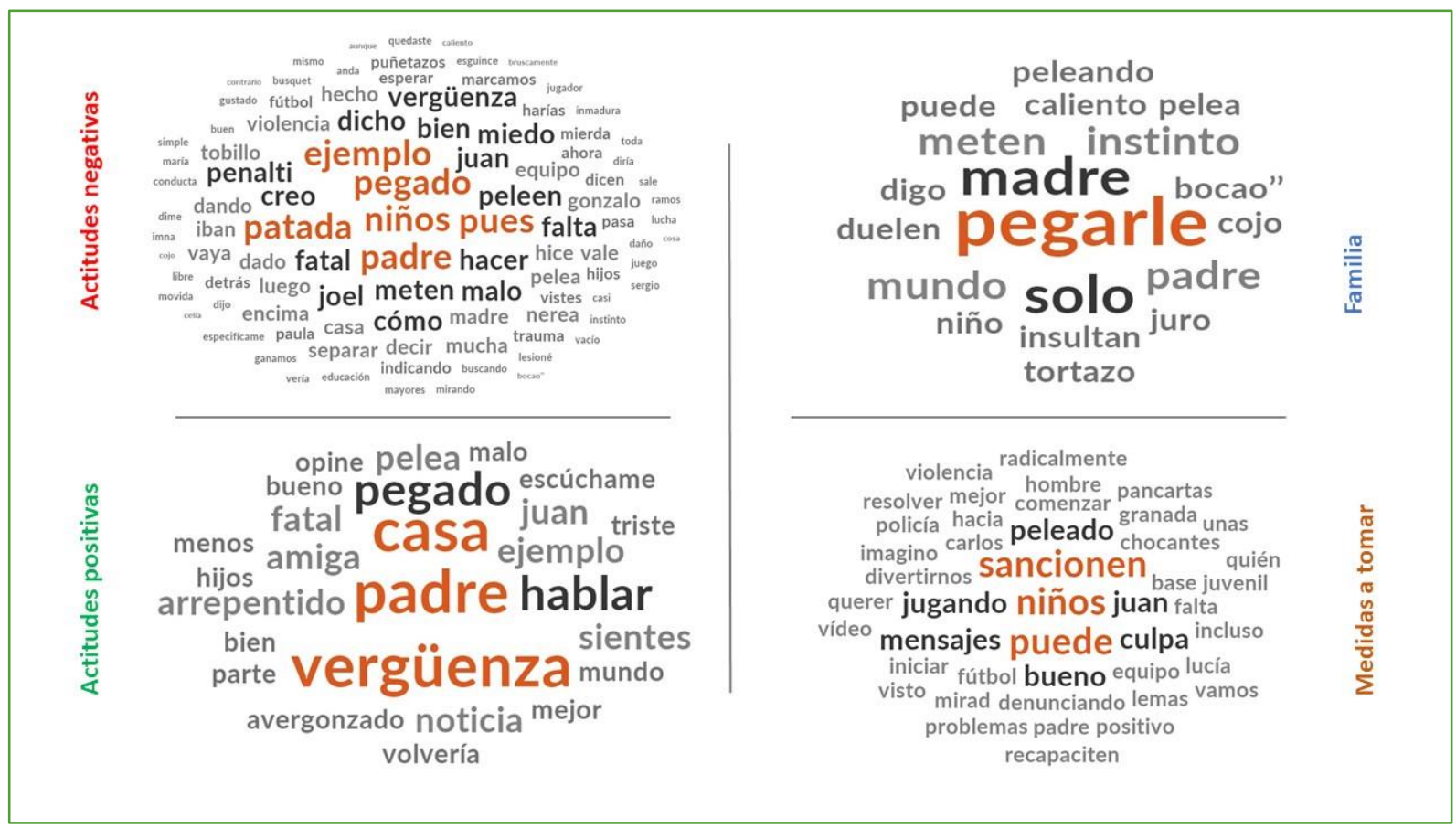

Figura 6 - Comparativa de conceptos en las categorías centrales.

Analizando los conceptos y su presencia en cada una de las categorías, vemos como la figura del padre aparece en las que se vinculan a las actitudes, 
tanto negativas como positivas y se identifica, aunque con escasa presencia en las medidas a tomar y la familia. Mientras, la madre solo aparece cuando hablamos de la familia. Lógicamente un concepto clave que se identifica en todas ellas es la relacionada con la agresión, en diferentes formas: pelea, pegar, bocao, etc. Es significativo que no se hable de las agresiones verbales, que parece son asumidas con mayor normalidad en el microsistema del fútbol.

Entrando en un análisis más discursivo, es significativo como Marcelo ${ }^{2}$ interviene sobre la pelea de los padres para dejar claro que desde su opinión «[los padres y las madres]no deberían de pelearse porque los niños son los que tienen que hablar», manifestando claramente que la resolución de los conflictos se debe hacer desde el diálogo y no desde la agresión verbal o física. En este tipo de respuestas es donde se evidencia el modelo de resolución de conflictos del centro educativo, en este caso basado en el diálogo (CAPLLONCH et al., 2018). Comentario que se ve reforzado en la misma línea, aunque con un nuevo matiz, por parte de Benzema, cuando reprocha a los padres que «Yo lo primero que hago es ir a ver si mi hijo está bien en vez de pelearme». Pero frente a este tipo de respuestas, también emerge la empatía de Varane que, olvidando el diálogo como punto de encuentro, deja salir su instinto primario y asegura que «Sí le pegaran a mi madre, yo te juro que le meto al tío. Aunque me peguen, es que yo lo caliento». Es en este tipo de declaraciones donde debemos poner el acento en las clases de Educación Física, aunque sea complejo parar las reacciones iniciales de los niños y niñas ante la agresión.

Para cerrar esta aproximación general a las claves del discurso, emerge un concepto clave: vergüenza. En este punto debiéramos preguntarnos quién debiera educar a quién. Como diría Busquets «Yo me sentiría mal, sentiría vergüenza de que mis padres hicieran eso». Es este tipo de reflexión lo que nos anima a seguir con la pedagogía del diálogo, porque comienzas a descubrir que la labor del día a día va calando en el bagaje valórico de nuestro alumnado.

Si nos queda claro desde este análisis, que los conceptos con mayor presencia en el alumnado son aquellos que hemos abierto anteriormente y se relacionan de forma clara con la vergüenza que sienten ante el comportamiento de estos padres. La presencia de la violencia en terrenos de juego que debieran ser espacios educativos y no contravalóricos y el miedo de verse en una situación que los supera porque no llegan a comprender, es un tema a resolver en los campos de fútbol de categorías inferiores. No se puede estar por la mañana en la escuela tejiendo valores sociales y por la tarde en el campo de fútbol destejiendo lo realizado con una formación en la que el fin siempre justifica los medios utilizados. Esta estrategia del manto de Penélope respecto a la actividad física y el

\footnotetext{
2 Se han utilizado nombres de jugadores de futbol como apodos, para ocultar el verdadero nombre de los niños y niñas participantes
} 
deporte no es sostenible desde un modelo educativo orientado hacia la justicia social.

Lo que hemos podido observar en el análisis realizado es la diferencia que se abre entre el discurso de los niños frente al de las niñas. En la figura 7 se aprecia perfectamente como en los niños hay un claro pico del discurso centrado en la violencia, exactamente esa es la mayor preocupación también de las niñas, pero se abre una diferencia clara, mientras que en ellos el resto del discurso es muy uniforme, salvo dos pequeños picos en la idea de la humillación y la familia, en ellas emergen con fuerza junto a la violencia, el mal ejemplo y la vergüenza que sienten ante la situación visionada.

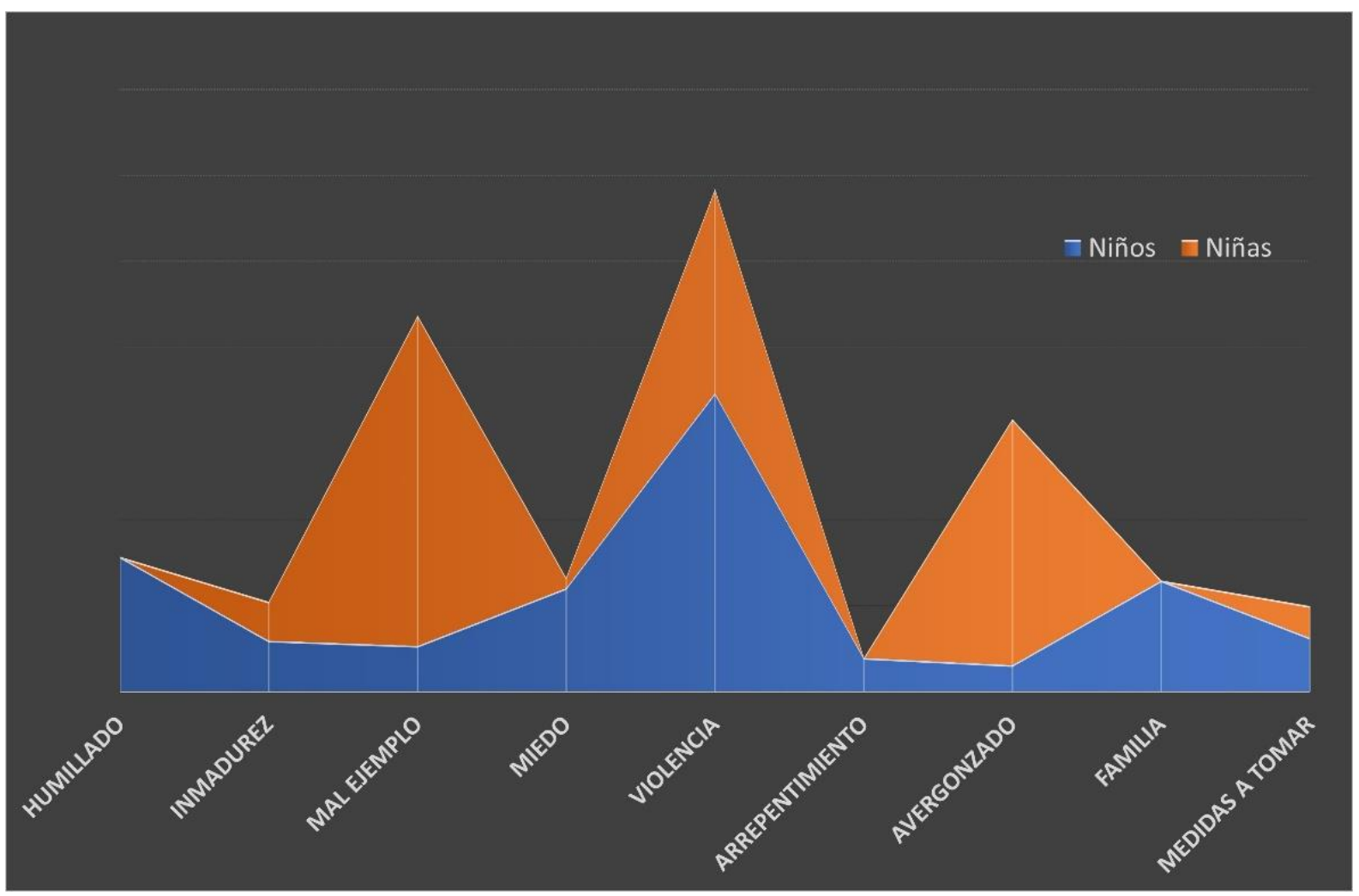

Figura 7 - Análisis de los discursos em función del sexo.

Si nos centramos en la violencia, los niños la asocian a la familia, especialmente a sus madres, lo que deja en evidencia unos hogares claramente patriarcales. Esto queda totalmente patente cuando Isco dice en la tertulia que «Maestra, yo si fuesen a pegarle a mi madre, yo me metería, es que te sale de dentro. Porque si a tu madre le pegan y le meten un tortazo, yo me metería». Es aquí cuando termina el diálogo y respondemos con la agresión violenta, lo que nos viene a decir que erradicar este tipo de actitudes en estos niños es una tarea que se antoja compleja. Pero si nos acercamos a los discursos de ellas, el patriarcado tan marcado en los niños se suaviza y se limitan a describir la situación que se está produciendo en el campo, sin llegar a su valoración. Piqué lo expresa desde la perspectiva mencionada y reconoce que no sabe «si unos niños habían perdido o que eran del equipo contrario, entonces los padres se han peleado porque ha 
dicho "mi niño es mejor"». Si reflexionamos sobre este punto lo que podemos apreciar es la diferencia existente en la implicación de ambos en el problema. Podría ser fruto del patriarcado que distancia a las niñas del deporte en general y del fútbol en particular, lo que hace que su percepción sobre lo acontecido, al estar enmarcado en un espacio de juego, lo consideren en cierta manera normalizado e incluso lógico en el ambiente en el que se desarrolla (CÁRDENAS-RODRÍGUEZ; TERRÓN-CARO; MONREAL-GIMENO, 2019).

Donde ambos coinciden en sus reflexiones es la sensación de vergüenza que se transmite en el incidente. Ni ellos ni ellas llegan a entender que estas cosas puedan ocurrir en un espacio de juego, así Iniesta manifiesta que se «sentiría mal, sentiría vergüenza de que mis padres hicieran eso» y por su parte Asensio va un poco más lejos y no solo se sentiría mal por lo ocurrido, sino que hablaría con sus padres y les expresaría que «está muy mal lo que ha hecho y que he sentido vergüenza y que eso no es un buen ejemplo».

Para finalizar esta breve exposición e interpretación de los resultados más destacados de la experiencia, queremos identificar la empatía que muestran algunos de los niños cuando constatan que lo que acaban de ver en el vídeo, es habitual en este deporte y en estas categorías, siendo poco o nada lo que se hace por corregirlo desde los estamentos correspondientes. Nos ha sorprendido las palabras de Bale, que nos narra un episodio vivido por él en una competición de benjamines en la que le «pegaron una patada por detrás que fue penalti, y como marcamos el penalti los padres de los otros niños me empezaron a decir que me iban a esperar después para pegarme». El traslado de la violencia que se hace del campo profesional al escolar es una constante en este deporte. En gran cantidad de ocasiones somos nosotros, los educadores de la corporeidad de los niños y niñas quienes debiéramos intervenir desde el ámbito escolar para invertir estas conductas que, si no agraden al cuerpo físico y se consideran parte del juego, si están menoscabando el cuerpo psicosomático de los niños y niñas. Siempre es fácil hablar de la educación integral desde la Educación Física, pero la realidad dista aún mucho de las buenas declaraciones de intenciones plasmadas en el currículum y su posterior desarrollo escolar del área.

\section{CONSIDERACIONES FINALES}

Vamos a cerrar esta reflexión sobre las posibilidades de educar el cuerpo desde una visión holística, a través del aprendizaje dialógico corporal y concretamente desde la tertulia dialógico corporal, planteándonos unas reflexiones finales que, a modo de interrogantes, queremos que sirvan de detonante para acometer un cambio profundo de la Educación Física Escolar.

Asistimos a una reproducción de las funciones de la Educación Física, que, aunque tratan de ser replanteadas desde el currículum hacia un sentido más Social 
y enlazado con los grandes temas que se plantean a nivel mundial, especialmente relacionados con la Justicia Social y el aprendizaje desde las experiencias con los otros. Pero la realidad nos dice que el aula de Educación Física ha cambiado poco o de forma muy aislada con experiencias puntuales. ¿Dónde estamos equivocándonos?, ¿Qué estamos haciendo mal? Quizás un replanteamiento de la formación inicial sería el punto de inicio, junto a la diseminación entre los actuales docentes de experiencias exitosas puesta en acción en el aula. Además, es preciso salir de la espiral productiva que nos impone una universidad cada vez más mercantilizada y competitiva y apostar por investigar con y para la escuela, buscando soluciones a problemas reales, dejando de crear nubes de algodón de azúcar desde una investigación irreal y descontextualizada, construida con la exclusiva finalidad de servir a nuestra individualidad, obviando el beneficio social.

Además, vemos como el currículum estatal y su desarrollo escolar se inundan de conceptos como reflexión y crítica, pero una vez más carecemos de herramientas que hagan visible estos conceptos en las aulas de la enseñanza obligatoria. ¿Es posible cambiar el rumbo y apostar por pedagogías más dialógicas que transfieran la responsabilidad y el placer de aprender a los estudiantes? Pensamos que sí, pero al mismo tiempo reconocemos que la presión social sobre la pérdida de los tiempos de actividad física en adolescentes, niñas y niños y la "pandemia" de la obesidad infantil, está provocando el regreso a modelos biomotrices que apuestan por cantidad en vez de por calidad.

Por último, entendemos importante que la educación Física se abra al diálogo y salga de los modelos directivos que la colonizan. ¿Es posible este cambio? Sólo si apostamos por cambiar la Educación en general, salimos de nuestro espacio particular y unimos nuestra voz a la del resto de docentes que creen que aún es posible educar para construir un mundo mejor. Entender que el cambio es político y no metodológico. Esta es la llave que puede abrir la puerta del cambio.

\section{NOTAS}

\section{CONFLITOS DE INTERESSE}

Os autores não têm conflitos de interesse, incluindo interesses financeiros específicos e relacionamentos e afiliações relevantes ao tema ou materiais discutidos no manuscrito.

\section{Autoria e CoAutoria}

A autoria é responsável pelos conteúdos do texto. Os autores declaram que participaram de forma significativa na construção e formação desde estudo, tendo, enquanto autor, responsabilidade pública pelo conteúdo deste, pois, contribuíram diretamente para o conteúdo intelectual deste trabalho e satisfazem as exigências de autoria. 
Enrique Rivera García - Concepção e desenvolvimento (desde a ideia para a investigação ou artigo, criou a hipótese); Desenho metodológico (planejamento dos métodos para gerar os resultados); Supervisão (responsável pela organização e execução do projeto e da escrita do manuscrito); Coleta e tratamento dos dados (responsável pelos experimentos, pacientes, organização dos dados); Análise / interpretação (responsável pela análise estatística, avaliação e apresentação dos resultados); Levantamento da literatura (participou da pesquisa bibliográfica e levantamento de artigos); Redação (responsável por escrever uma parte substantiva do manuscrito); Revisão crítica (responsável pela revisão do conteúdo intelectual do manuscrito antes da apresentação final).

Carmen Trigueros-Cervantes - Concepção e desenvolvimento (desde a ideia para a investigação ou artigo, criou a hipótese); Desenho metodológico (planejamento dos métodos para gerar os resultados); Supervisão (responsável pela organização e execução do projeto e da escrita do manuscrito); Coleta e tratamento dos dados (responsável pelos experimentos, pacientes, organização dos dados); Análise / interpretação (responsável pela análise estatística, avaliação e apresentação dos resultados); Levantamento da literatura (participou da pesquisa bibliográfica e levantamento de artigos); Redação (responsável por escrever uma parte substantiva do manuscrito); Revisão crítica (responsável pela revisão do conteúdo intelectual do manuscrito antes da apresentação final).

Francisco Javier Giles Girela - Concepção e desenvolvimento (desde a ideia para a investigação ou artigo, criou a hipótese); Desenho metodológico (planejamento dos métodos para gerar os resultados); Supervisão (responsável pela organização e execução do projeto e da escrita do manuscrito); Coleta e tratamento dos dados (responsável pelos experimentos, pacientes, organização dos dados); Análise / interpretação (responsável pela análise estatística, avaliação e apresentação dos resultados); Levantamento da literatura (participou da pesquisa bibliográfica e levantamento de artigos); Redação (responsável por escrever uma parte substantiva do manuscrito); Revisão crítica (responsável pela revisão do conteúdo intelectual do manuscrito antes da apresentação final).

\section{REFERÊNCIAS}

ÁGUILA, Cornelio; LÓPEZ, Juan José. Cuerpo, Corporeidad y Educación: Una mirada reflexiva desde la educación. Retos. Nuevas Tendencias En Educación Física, Deporte y Recreación, v. 35, p. 413-421, 2019.

AUBERT, Adriana; GARCÍA, Carmen; RACIONERO, Sandra. El Aprendizaje Dialógico. Cultura y Educacion, v. 21, p. 129-139, 2009.

BARBERO, José Ignacio. La Escolarización Del Cuerpo: reflexiones En Torno a La Levedad de Los Valores Del Capital "Cuerpo" en Educación Física. Revista Iberoamericana de Educación, v. 39, p. 25-52, 2005.

CAPLLONCH, Marta; FIGUERAS, Sara; CASTRO, Marcos. Conflict Resolution Strategies in Physical Education. Apunts. Educacion Fisica y Deportes, p. 50-67, 2018.

CÁRDENAS-RODRÍGUEZ, Rocio.; TERRÓN-CARO, Teresa; MONREAL-GIMENO, María del Carmen. Educación Primaria y alumnas gitanas. Análisis de las barreras sociales en contextos de exclusión. Revista de Investigación Educativa, v. 37, n. 1, p. 75-91, 2019. 
CARRASCO-SEGOVIA, Sara; HERNÁNDEZ-HERNÁNDEZ, Fernando. Cartografiar los afectos y los movimientos en el aprender corporeizado de los docentes'. Movimento, $v$. 26, p. $1-13,2020$.

CASTRO-SANDÚA, Marcos, GÓMEZ-GONZÁLEZ, Aitor; MACAZAGA-LÓPEZ, Ana. Aprendizaje Dialógico y Grupos Interactivos en Educación Física. Retos: Nuevas Tendencias En Educación Física, Deporte y Recreación, v. 25, p. 174-179, 2014.

DEVÍS-DEVÍS, José; MOLINA, José. Las Funciones de La Educación Física Escolar: De La Modernidad a La Postmodernidad'. In: DE ANDRADE; CAPARROZ. Educaçao Física Escolar: Política, Investigaçao e Intervençao. São Paulo: LESEF/UFFS, 2004. p. 35-49

DEVÍS-DEVÍs, José. Los Discursos Sobre Las Funciones de La Educación Física Escolar Continuidades, Discontinuidades y Retos. Valencia: Universidad de Valencia, 2018.

FERNÁNDEZ-ENGLER, Rodrigo. Cobardes, 2017. Disponible en: https://www.youtube.com/watch?v=VOjs4j3bQhc\&feature $=e m b$ rel end. Acceso en: 15 mayo 2020.

FERREYRA, Ángela María. La Transformación Soñada: De Un Centro Rural Agrupado a Una Comunidad de Aprendizaje. 2016. Tesis (Doctoral) - Facultad de Educación, Universidad de Valladolid, 2016.

FLECHA, Ramón. Cambio, Inclusión y Calidad En Las Comunidades de Aprendizaje. Cultura y Educación, v. 21, p. 157-170, 2009.

FREIRE, Paulo, Pedagogía Del Oprimido. Madrid: Siglo XXI, 1970.

GILES-GIRELA, Francisco Javier; RIVERA-GARCÍA, Enrique; TRIGUEROS-CERVANTES, Carmen. Evaluación de la dedicación de los estudiantes universitarios al aprendizaje desde el análisis del diario digital. Arquivos em movimento, Rio de Janeiro, v.16, n.2, p. 123-142, jul./dez. 2020.

HABERMAS, Jürgen. Teoría de La Acción Comunicativa, Madrid: Taurus, 1987.

MARTÍNEZ-ÁLVAREZ, Lucio. El Tratamiento de Lo Corporal en la Formación Inicial del Profesorado. Revista Interuniversitaria de Formación Del Profesorado, v. 27, p. 161-175, 2013.

MORENO-DOÑA, Alberto; CAMPOS-VIDAL, Marlen; ALMONACID-FIERRO, Alejandro. Las Funciones de La Educación Física Escolar: Una Mirada Centrada en la Justicia Social y la reconstrucción del conocimiento. Estudios Pedagógicos, v. 38, p. 13-26, 2012.

RIVERA-GARCÍA, Enrique; SÁNCHEZ-LÓPEZ, Mónica; GILES-GIRELA, Francisco Javier. Las tertulias pedagógicas aplicadas al ámbito de la Educación Física: una propuesta desde el aprendizaje-servicio universitario. In: CHIVA-BARTOLL; O.; PALLARES-PIQUER, M. Aprenzijage-servicio: pasaporte para un futuro mejor. Sevilla: Egregios, 2018. p. 3552.

RODRÍGUEZ-PALACÍN, Jesús. La Educación Física Como Factor Importante Para Combatir El Bulling en los centros escolares, 2017 Tesis (de Grado) - Facultad de Educación, Universidad de Granada, 2020.

RODRÍGUEZ, Rosana. La Vida Encarnada: Significaciones sobre la Experiencia Corporal de las Mujeres. Journal for Educators, Teachers and Trainers, v. 5, p. 115-128, 2014. 
SÁNCHEZ-LÓPEZ, Mónica, Las Tertulias Dialógicas aplicadas al ámbito de la Educación Física, 2017 Tesis (de Grado) - Facultad de Educación, Universidad de Granada, 2017.

SERRANO, Ángeles; MIRCEVA, Jasmina; LARENA, Rosa. Dialogic Imagination in Literacy Development. Psicodidáctica, v. 15, p. 191-205, 2010.

TRIGUEROS-CERVANTES, Carmen; RIVERA-GARCÍA, Enrique; RIVERA-TRIGUEROS, Irene. The Use of NVivo in the Different Stagesof Qualitative Research. In: INTERNATIONAL SYMPOSIUM ON QUALITATIVE RESEARCH, 2. 2017. Proceedings... Lisboa: CIAIQ, 2017. v. 5. p. 80-1.

TURNER, Bryan. Los Avances Recientes En La Teoría Del Cuerpo, Reis, v. 11, p. 11-40, 1994.

Artigo submetido ao sistema de similaridade Turnitin $®$.

A revista Conexões utiliza a Licença Internacional Creative Commons Atribuição 4.0, preservando assim, a integridade dos artigos em ambiente de acesso aberto.

A Revista Conexões é integrante do Portal de Periódicos Eletrônicos da Unicamp e associado/membro das seguintes instituições:
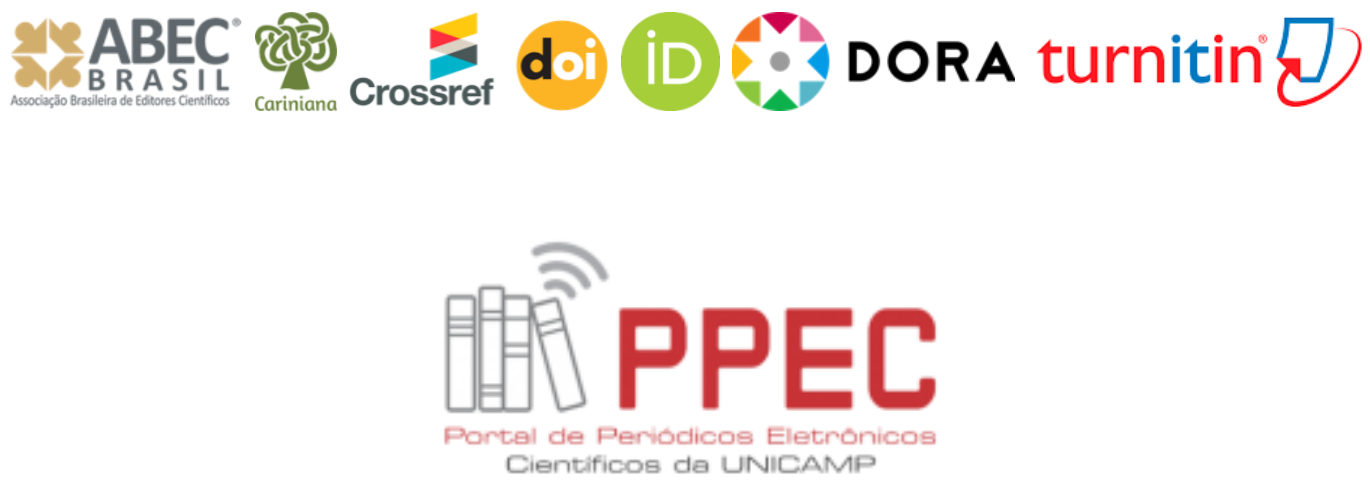